\title{
Ultimate Strength of Cylindrical Shells with Cutouts
}

\section{Sang Eui Lee ${ }^{1}$, Selcuk Sahin ${ }^{2,3}$, Philippe Rigo ${ }^{2}$, Minsue Park ${ }^{4}$ and Jeom}

Kee Paik ${ }^{1,4,5^{*}}$

${ }^{1}$ The Korea Ship and Offshore Research Institute (The Lloyd's Register Foundation Research Centre of Excellence), Pusan National University, Busan 46241, Republic of Korea

${ }^{2}$ Naval Architecture and Transport Systems Department (LHCN), University of Liège, 6 Quai Banning, B4000 Liège, Belgium

${ }^{3}$ Laboratoire d'Hydrodynamique, Energétique et Environement Atmosphérique (LHEEA), Ecole Centrale de Nantes, 44321 Nantes, France

${ }^{4}$ Department of Naval Architecture and Ocean Engineering, Pusan National University, Busan 46241, Republic of Korea

${ }^{5}$ Department of Mechanical Engineering, University College London, Torrington Place, London WC1E 7JE, UK

*Corresponding author. Tel.: +82 51 510-2429.

Email address: jeompaik@ pusan.ac.kr (J.K. Paik) 


\section{Ultimate Strength of Cylindrical Shells with Cutouts}

Cutouts - perforations that are often made in wind turbine towers to allow access or passage - can also reduce the towers' ultimate strength. Thus, cutouts may need to be included in the ultimate strength formulations for wind turbine towers as an influential parameter, where significant. The aims of this study are to examine the effects of cutouts on the ultimate-strength characteristics of wind turbine towers and to propose some practical design formulae to predict the reduced ultimate strength. The structural features of cutouts and towers in real wind turbines are investigated, and the effects of different design variables - such as shape, location, aspect ratio, column slenderness ratio, and column aspect ratio - on the ultimate-strength behaviour are described. The ultimate strengths of the towers are computed using elastic-plastic large-deflection finite element analyses. Practical design formulae accommodating a whole range of actual dimensional characteristics of cutouts and towers are derived and proposed. The findings of this research and the proposed design formulae have the potential to enhance the structural design and safety assessment of wind turbine towers.

Keywords: Cutouts, ultimate strength, wind turbine tower, parameters of influence, nonlinear finite element method 


\section{Nomenclature}

A Area of the cutout

$b \quad$ Breadth of the cutout

$D\left(D_{\max } / D_{\min }\right)$ Diameter (maximum/minimum) of the wind turbine tower

E Elastic modulus of the material

$F_{R} \quad$ Reference force of the wind turbine tower

$F_{\text {Tower }} \quad$ Force acting on the wind turbine tower

$F_{u} \quad$ Ultimate force of the wind turbine tower

$F_{\text {wind }} \quad$ Thrust induced by blades

$F_{z} \quad$ Load in $z$-axis

$h \quad$ Height of the cutout

$h_{o} \quad$ Distance from the lower end to the centre of the cutout

$H \quad$ Height of the wind turbine tower

$H_{s} \quad$ Height of 1 st section

M Pure bending moment

$M_{u} \quad$ Ultimate bending moment

$M_{P} \quad$ Plastic bending moment

$M_{y} \quad$ Pure bending moment in $y$-axis

$r \quad$ Radius

$R \quad$ Corner radius of the cutout

$\mathrm{R}^{2} \quad$ Adjusted R-square

$t\left(t_{\max } / t_{\text {min }}\right) \quad$ Thickness (maximum/minimum) of the wind turbine tower

$t_{c} \quad$ Thickness of the cutout 
$T \quad$ Torque moment

$u_{x}, u_{y}, u_{z} \quad$ Translational restraints in the $x$-, $y$ - and $z$-axis

$w_{o} \quad$ Initial imperfection

W Weight

$\alpha \quad$ Aspect ratio (height to breadth) of the cutout

$\beta \quad$ Slenderness ratio (breadth to thickness) of the cutout

$\delta_{\max } \quad$ Maximum deformation

$\gamma \quad$ Column aspect ratio (height to diameter) of the wind turbine tower

$\gamma_{\max } / \gamma_{\min } \quad$ Maximum/minimum column aspect ratio (height to maximum/minimum diameter) of the wind turbine tower

$\lambda$ Column slenderness ratio (diameter to thickness) of the wind turbine tower $\lambda_{\max } / \lambda_{\min } \quad$ Maximum/minimum column slenderness ratio (maximum/minimum diameter to maximum/minimum thickness) of the wind turbine tower

$v \quad$ Poisson's ratio

$\theta \quad$ Angle of the cutout in the circumferential direction

$\theta_{x}, \theta_{y}, \theta_{z} \quad$ Rotational restraint in the $x$-, $y$ - and $z$-axis

$\sigma \quad$ Coefficient of correlation

$\sigma_{Y} \quad$ Yield stress of the material

$\xi_{D}, \xi_{t}, \xi_{h}, \xi_{b}, \xi_{C} \quad$ Coefficients of design formula for axial compression

$\zeta_{D}, \zeta_{t}, \zeta_{h}, \zeta_{b}, \zeta_{C} \quad$ Coefficients of design formula for pure bending 


\section{Introduction}

Cutouts are widely used in steel structures to provide access to or lighten the structure. It is no wonder, then, that these perforations reduce both the buckling and ultimate strength of such structures. In particular, wind turbines, which have relatively large doors, can be exposed to considerable strength reduction that could result in significant structural failure in their towers. It is thus of great importance to develop advanced technologies that can predict a tower's reduced strength using the size of the cutout.

The regulations for designing reliable and safe wind turbines have been developed and recommended by various authorities (ECCS 1980, DIN 18800-4 1990, EN1993-1.6 2006, DNVGL 2013a, DNVGL 2013b). However, there are no detailed guidelines for predicting the reduced strength of towers.

It is noted that there have been far fewer useful research attempts to investigate the effects of cutouts on the structural capacity of circular cylindrical shells, than those investigating plates (Sabir and Chow 1983, Brown and Yettram 1986, Azizian and Roberts 1983, Shangmugam et al. 1999, Durban and Zuckerman 1999, Betten and Shin 2000, El-Sawy et al. 2004, Paik 2007, Kim et al. 2009, Wang et al. 2009). For a couple of decades, a number of studies were conducted to provide buckling analyses of circular cylindrical shells (Brazier 1927, Reissner 1961, Seide and Weingarten 1961, Fabian 1977, Gellin 1980) with cutouts under axial compression (Schenk and Schuëller 2007, Shariati and Rokhi 2010, Ghazijahani et al. 2015) and pure bending (Yeh et al. 1999, Dimopoulos and Gantes 2012, 2013, 2015, Guo et al. 2013, Dimopoulos et al. 2015).

The aims of this study are to use nonlinear finite element analysis to examine the effect of cutouts on the ultimate-strength characteristics and to propose simple formulae to estimate the reduced ultimate strength of wind turbine towers under axial 
compression and pure bending. The structural features of wind turbines are investigated using data collected from 102 wind turbines in service. Finite element modelling techniques are developed to calculate the ultimate-strength behaviour of the towers with a variety of design variables, such as cutout shapes and locations, aspect ratios, column slenderness ratios and column aspect ratios. The developed nonlinear finite element method modelling is then validated. For the parametric series analyses, a design of experiment (DOE) method, such as central composite design (CCD), is applied. Numerical computations are then used to derive a plausible design formula that predicts the ultimate strength of the towers.

\section{Literature Review}

In the early days, buckling analyses of circular cylindrical shells subjected to pure bending and axial compression were first conducted by analytical and experimental manners. In particular, Brazier (1927) noted that the ultimate strength is directly related to the ovalisation of the tube cross-section under bending, and thus derived an expression for the strain energy per unit tube length in terms of the change in axial curvature. Reissner (1961) further developed a more general formulation for thin-walled cylindrical shells of arbitrary cross-sections. Seide and Weingarten (1961) used a modified Donnell equation and the Galerkin method and found that the maximum elastic buckling stress under bending is equal to the critical compressive stress under axial compression. Sherman (1976) experimentally identified that shells with a column slenderness ratio where $\lambda$ was greater than about 50 did not have sufficient plastic hinge rotation capacity to develop the classical ultimate strength. Fabian (1977) observed two modes of failure of infinitely long cylindrical elastic shells subjected to bending, pressure and axial loads, and found that the circumferential flattening that 
constituted an ultimate load and compression wrinkles generated bifurcation buckling axially. Gellin (1980) demonstrated how extending the results of Brazier (1927) into the plastic range confirmed the results of limit states observed by Fabian (1977).

Traditionally, experimental tests have been regarded as the most efficient way of obtaining technical solutions, despite their high costs. Over the past 50 years, computing speeds and the capabilities of numerical tools have improved significantly. Hence, the contributions made by numerical simulations to engineering applications are increasing. The same trend has been observed in the wind turbine industries, and extensive experimental tests and numerical simulations have been conducted to examine the loadcarrying capacity of circular cylindrical shells with cutouts under axial compression and pure bending.

For axial compression, Tennyson (1968) experimentally observed membrane stress distributions and isoclinic patterns around the edges of cutouts using photoelastic shells. Jullien and Limamto (1998) found that the buckling strength is sensitive to a cutout's angle or circumferential size, based on parametric studies of its shape (square, rectangular, circular) and dimensions (axial and circumferential sizes, diameter). Furthermore, they pointed out the importance of initial imperfections for numerical simulations. Schenk and Schuëller (2007) studied the effects of random geometric imperfections on the critical load of thin-walled cylindrical shells under axial compression with rectangular cutouts. They found that the coefficient of variation of the critical load did not decrease with the imperfections' magnitude. Han et al. (2006) observed that the location and size of a cutout significantly affect the buckling load; specifically, cutouts located near the fixed boundary could effectively absorb energy and redistribute the load more efficiently. Shariati and Rokhi (2008 2010) reported that longer shells exhibit much more sensitivity to the positions of the cutouts. Moreover, 
they observed that the buckling strength decreases as height increases with a constant cutout width. Ghanbari Ghazijahani et al. (2015) experimentally found a symmetric ring-shaped bulging wave in an intact specimen after initiating buckling. They noted that the effects of the cutouts' heights on the capacity were less than 5\% under axial compression.

For pure bending, Kyriakides and Ju (1992) and Ju and Kyriakides (1992) observed that thinner shells developed short wavelength periodic ripples on the compressed sides of the shells, and the shells buckled locally and collapsed soon after the ripples appeared. In contrast, thicker shells were found to exhibit limited load instability as a direct consequence of the ovalisation of the shell cross-section caused by pure bending. Yeh et al. (1999) observed that for a shell with a circular cutout, the ultimate strength decreased as the diameter of the cutout increased, whereas for a shell with a rectangular cutout, the ultimate strength decreased as the size of the cutout increased. It was also found that the ultimate strength of a shell with a cutout on the compression side was less than for a cutout on the tension side, and the ultimate strength increased when a cutout was close to the end of the clamped shell. Guo et al. (2013) found that an increase in the $D / t$ ratio resulted in a more pronounced local buckling phenomenon, with the stiffeners increasing the load carrying capacity while improving the ductility.

The most distinguished numerical and experimental works were Dimopoulous's series of studies (Dimopoulos and Gantes 2012, Dimopoulos and Gantes 2013, Dimopoulos and Gantes 2015, Dimopoulos et al. 2015) on circular cylindrical shell structures. Experimental and numerical studies of the buckling behaviour of cantilevered circular cylindrical shells with cutouts and stiffening were conducted, and it was confirmed that the presence of the cutouts led to a reduction in strength. The 
lowest collapse load appeared when a cutout was situated on the compression side (Dimopoulos and Gantes 2012). It was also shown that simple stiffening types, consisting of either a peripheral frame or two longitudinal stiffeners with a ring, were particularly efficient and could be used instead of more complex examples (Dimopoulos and Gantes 2013). The importance of geometrical and material nonlinearities, including initial imperfections, was also noted (Dimopoulos and Gantes 2015). Finally, the stiffening effects of cutouts on circular cylindrical shells under dynamic wind loading were assessed using aeroelastic code. It was concluded that the dynamic effect led to a small decrease in tower strength compared to the effect obtained via static analysis, but this reduction was less than $10 \%$ in all investigated cases (Dimopoulos et al. 2015).

\section{Structural Features}

\subsection{Definition of geometrical parameters}

Wind turbines typically consist of circular cylindrical shell sections connected by bolted flanges, as shown in Fig. 1. The geometrical attributes of a typical wind turbine tower with a cutout are defined here. The following four parameters for wind turbine towers are considered: minimum column aspect ratio $\left(\gamma_{\min }=H / D_{\min }\right)$; maximum column aspect ratio $\left(\gamma_{\max }=H / D_{\max }\right)$; minimum column slenderness ratio ( $\left.\lambda_{\min }=D_{\min } / t_{\min }\right)$; and maximum column slenderness ratio $\left(\lambda_{\max }=D_{\max } / t_{\max }\right)$. The other two cutout parameters considered are the aspect ratio $(\alpha=h / b)$ and the slenderness ratio $\left(\beta=b / t_{c}\right)$.

\subsection{Geometrical features}

Data on 102 wind turbines and their cutouts were collected where the capacities ranged from 0.5 to 5.0 MW. The principal features are displayed in Appendix Table A1, 
and the geometrical characteristics of each parameter predefined in Section 3.1 are analysed. The statistical distribution of the parameters is shown in Figs 2 and 3. Table 1 summarises the range and most probable values of each parameter. These findings are then used to identify the geometrical parameters of the standard wind turbine tower and the cutout: $H=65,000 \mathrm{~mm} ; D_{\max }=3,750 \mathrm{~mm} ; t_{\max }=30 \mathrm{~mm} ; h=1,900 \mathrm{~mm} ; b=700$ $\mathrm{mm}$; and $t_{c}=30 \mathrm{~mm}(\alpha=2.875, \beta=25.0)$. Based on those parameters, the dimensions of the flanges are designed and illustrated in Table 2 (Sahin 2015). It was assumed that the standard wind turbine tower is composed of 3 sections, as shown in Fig. 4, and that the height of the first section used in the present study was $H_{S}=12,655 \mathrm{~mm}$. Hereafter, because the tower section applied in the present study had a uniform thickness, $t$, the thickness of the cutout $\left(t_{c}\right)$ and the maximum thickness of the wind turbine tower $\left(t_{\max }\right)$ are represented as the thickness of the wind turbine tower $-t\left(=t_{c}=t_{\max }\right)-$ and the diameter of the tower section $(D)$ is the same as the maximum diameter of the wind tower $\left(D_{\max }\right)$.

\subsection{Selection of parameters}

CCD is an experimental design for building a second-order model without needing to use a complete 3-level factorial experiment. The CCD with the fractional factorial design was used in this study as a deterministic method for parameter selection. The locations of the design points were determined based on the number of input parameters, in accordance with the DOE method. The example points of the CCD with three input parameters are shown in Fig. 5. 


\subsection{Reference capacity of circular cylindrical shells without the cutout}

In the section on wind turbine structures with cutouts, the first yield occurred near the cutout where the highest compression developed and rapidly expanded around the cutout with further loading. The entire load-carrying capacity of the wind turbine structure with the cutouts depended on the geometrical dimensions and material properties. In the present study, the reference buckling loads of the shells without cutouts subjected to axial compression (Shariati and Rokhi 2008) and pure bending moments (Dimopoulous and Gantes 2013) were defined as:

$$
\begin{aligned}
& F_{R}=\pi D t \times \sigma_{Y} \\
& M_{P}=\frac{4}{3} \pi\left(\left(r+\frac{t}{2}\right)^{3}-\left(r-\frac{t}{2}\right)^{3}\right) \times \sigma_{Y}
\end{aligned}
$$

where $F_{R}$ is the reference load of a wind turbine, $M_{P}$ is the plastic bending moment of a wind turbine, $D$ is the diameter of the wind turbine tower, $r$ is the radius of the wind turbine tower, $t$ is the thickness of the wind turbine tower and $\sigma_{Y}$ is the yield stress of the material.

\section{Nonlinear Finite Element Modelling}

\subsection{Finite-element model}

In this study, nonlinear finite element analysis is performed using ANSYSWorkbench (2015) to accommodate both geometrical and material nonlinearities. To solve highly nonlinear problems, including the very unstable snap-through response of shell structures, nonlinear stabilisation - which uses the line search method together with auto time stepping - is used. 
The SHELL181 element, which has four nodes with 6 degrees of freedom at each node, is used to model circular cylinder shells and the SOLID185 element, which has 8 nodes with 3 degrees of freedom at each node, is used to model the ring frame located at both ends of the circular cylinder shell section. The wind turbine is modelled on the result of the quasi-static material test of mild steel grade A, as shown in Fig. 6.

As in previous studies (Jullien and Limamto 1998, Schenk and Schuëller 2007, Dimopoulos and Gantes 2015), the effect of initial imperfection is properly applied. The maximum magnitude of initial deflection $w_{o}$ is assumed to be $30 \%$ of the thickness of the wind turbine tower; that is, $w_{o}=0.3 t$. The eigenvalue buckling mode is used to determine the shape of the initial deflection. Fig. 7 provides examples of the smallest buckling mode near the cutout, obtained from the eigenvalue buckling analyses for intact (no opening) and cutout under axial compression and pure bending. In order to maintain the simplicity of finite element method computations, the residual stress caused by welding is not considered in the present study.

\subsection{Loading conditions}

The loading regimes of wind turbines during operation are extremely complex. A proper understanding of loads on wind turbines and their structural responses are crucial to avoiding their catastrophic failure. In general, the types of loads acting on wind turbines in service can be classified into five categories: static, cyclic, stochastic, aerodynamic and mechanical. As shown in Fig. 8, the schematic free-body diagram of a wind turbine structure can be represented as three loads: a torque due to the blades, an axial force due to gravity and a bending moment due to the thrust of the blades and the transverse force on a tower.

In the present study, it is assumed that an axial force and bending moment are closely related to the wind turbine tower's failure or collapse. They are applied in an 
isolated manner rather than in combination to investigate the effect of each load on the ultimate strength of the wind turbine tower with a cutout. When it is essential to predict the ultimate strength accurately, the combined loads should be considered.

\subsection{Boundary conditions}

The boundary conditions investigated in this study are described in Fig. 9. The coordinate system used for their measurement is shown in Fig. 9(a). The restraints are described in detail below.

- Fixed boundary condition, as shown in Fig. 9(b)

- Bottom surface: translational restraints in the $x$-, $y$ - and $z$-directions, $u_{x}=u_{y}=u_{z}=0$; rotational restraint in the $x$-, $y$ - and $z$-direction, $\theta_{x}=\theta_{y}=\theta_{z}=0$.

As mentioned, a wind turbine with a cutout - as detailed in Section 3.2 - is regarded as being subject to axial compression in the z-axis and pure bending in the $y$ axis, as shown in Fig. 9(c).

\subsection{Mesh-convergence study}

This section presents the results of the mesh convergence study for 6 types of element sizes under pure bending when $\sigma_{Y}=299 \mathrm{MPa}$ and $w_{o}=0.3 t$. In the mesh convergence study, 6 element sizes are tested under pure bending. The ultimate strength is summarised in Fig. 10, and it is found that approximately 35,000 elements (F5, size $=$ $40 \mathrm{~mm}$ ) are sufficient to estimate the ultimate bending moment of the wind turbine. The authors assume that the mesh convergence for axial compression may agree with the results for pure bending.

\subsection{Validation}

The finite element modelling techniques developed in the present study are 
validated by the experimental results under axial compression (Ghanbari Ghazijahani et al. 2015) and pure bending (Dimopoulos and Gantes 2012). Fig. 12 shows the results of a validation study for the models shown in Fig. 11. It confirms that the developed finite element modelling technique is generally effective for simulating the ultimate strength of the wind turbine towers under axial compression and pure bending.

\section{Effects of Variables}

In this section, 3 sets of parametric studies of the results are presented. First, to investigate the effect of a cutout shape on the ultimate strength, 3 shapes - rectangular, elliptical and half-elliptical-rectangular - are considered. Second, to examine the effect that a cutout location has on the ultimate strength in the vertical and circumferential directions, 5 locations in the vertical direction and 9 in the circumferential direction are considered. Third, the cutout shape, aspect ratio, column slenderness (diameter to thickness) ratio and column aspect (height to diameter) ratio are taken as the design variables and their effects on the ultimate strength are widely calculated. To identify the combined effects of these variables on the ultimate strength, a DOE with the CCD method is applied for the selection of design points for a given range of each parameter from Section 3.2.

\subsection{Effects of cutout shape}

As stated earlier, previous researchers (Julien and Limam 1998, Yeh et al. 1999) attempted to examine the effects of cutout shape on the load-carrying capacities of circular cylindrical shells. They concluded that the existence of cutouts alters the nature of the moment-end-rotation response under pure bending. However, the effects of cutout shape on the load-carrying capacities were weak, and sometimes negligible.

The authors attempted to improve their understanding of the effects of cutout 
shape on the ultimate strength using the standard model predefined in Section 3.2. 3 types of shapes - rectangular, elliptical and half-rectangular-elliptical - were considered. To perform an accurate comparison, the area of each cutout, $A$, was kept the same. The parameters considered in this study are as follows:

- Shape: intact (no-opening), rectangular, elliptical, half-rectangular-elliptical

- Loading condition: axial compression, pure bending

The applied geometries are illustrated in Fig. 13, and their dimensions for the cutouts are summarised in Table 2. Fig. 14 describes a comparison of the load-carrying capacity with the no-opening model. The reduction rate of the ultimate strength of each shape for both loading conditions appeared to be around $80 \%$ intact. The present results confirm the previous findings (Julien and Limam 1998, Yeh et al. 1999) that the effect of shape is negligible.

Figs. 15 and 16 illustrate von Mises stress contours at various loading stages for the standard wind turbines with the rectangular cutout under axial compression and pure bending as an example. It was found that the stress concentration is initiated regions around the cutout and it increases with the further load. As it increases, the level of stress rapidly rises so that these regions yield before the tower reaches the buckling state. Finally, tower shell around the cutout buckles. It was surmised that the local buckling occurs at first around the cutout and then the tower buckling follows.

\subsection{Effects of cutout location}

The effects of cutout location in the vertical or circumferential directions were previously investigated by a number of researchers (Kyriakides and Ju 1992, Ju and Kyriakides 1992, Yeh et al. 1999, Han et al. 2006, Dimopoulos and Gantes 2012), who noted that as the cutout location neared the boundaries, the ultimate strength increased under axial compression. Moreover, the ultimate strength of a circular cylindrical shell 
with a cutout on the compression side was smaller than that of one with a cutout on the tension side under pure bending (Kyriakides and Ju 1992, Ju and Kyriakides 1992, Yeh et al. 1999, Dimopoulos and Gantes 2012). In order to assess the ultimate strength of circular cylindrical shells with cutouts, a series of nonlinear finite element method computations are performed for various cutout locations in the vertical and circumferential directions.

\subsubsection{Vertical direction}

To investigate the effects of cutout location in the vertical direction on the ultimate strength, the thickness, $t$, is kept the same at $30 \mathrm{~mm}$. 50 cases of series analyses were performed, and the parameters considered are as follows:

- Shape: elliptical

- Location in vertical direction, $h_{o} / H_{s}: 0.1,0.2,0.3,0.4,0.5$

- Column slenderness ratio, $\lambda=D / t: 90,110,125,130,150$

- Loading condition: axial compression, pure bending

Fig. 17 displays an example of applied geometries with varying cutout locations in the vertical direction, $h_{o} / H_{S}=0.1 \sim 0.5$.

Figs 18 and 19 illustrate the force-displacement and moment-rotation histories for various column slenderness ratios. It was found that the closer the cutout is to the fixed boundary, the more the ultimate strength increases in both loading conditions. However, while numerical results of cutouts located near the fixed boundaries measured the higher ultimate strength than others, a sudden drop in strength appears after the ultimate state for all cases in axial compression and some cases in pure bending. Although the closest cutout reveals the highest ultimate strength, it can not be regarded as the safest wind turbine in terms of structural integrity. It indicates that current 
locations of the cutout in the vertical axis may not be the optimum location in terms of safety and structural integrity. Therefore, it is recommended that when structural engineers determine the location of the cutout in the vertical direction, they must carefully consider it as one of crucial design aspects and have a design solution for enhancing structural capacity.

Fig. 20 summarises the non-dimensionalised load-carrying capacity, which varies with the cutout location in the vertical direction. The ultimate strength increases almost linearly as a function of the cutout location, and as the column slenderness ratio decreases, the reduction rate increases under both loading conditions. Moreover, the ultimate strength under pure bending is not sensitive to the variations in the column slenderness ratio; namely, increasing diameter except for $\lambda=150$.

\subsubsection{Circumferential direction}

The thickness, $t$, is kept the same at $30 \mathrm{~mm}$ under pure bending to identify the effects that cutout location in the circumferential direction has on the ultimate strength because the structural response under axial compression is symmetrical. 90 cases of series analyses are performed in total, and the parameters considered are as follows:

- Shape: half-rectangular-elliptical

- Column slenderness ratio, $\lambda=D / t: 125$

- Cutout angle, $\theta: 0,15,30,45,60,90,120,150,180$ degree

- Loading condition: pure bending

Fig. 21 illustrates an example of applied geometries with varying cutout angles in the circumferential direction, $\theta=0 \sim 180$ degrees.

Fig. 22 presents an example of moment-rotation histories with varying cutout angles and the non-dimensionalised load carrying strength. It confirms that when a cutout is located on the compression side ( $\theta=0$ degrees), the ultimate strength is 
minimal, and as the cutout angle increases, so do the ultimate strength. It is observed that the ultimate strength increases almost linearly until $\theta=90$ degrees, whereas it appears almost constant over 90 degrees ( $\theta=120,150,180$ degrees).

\subsection{Combined effects of aspect, column slenderness, and column aspect ratios}

To examine the effects of the aspect, column slenderness and column aspect ratios on the ultimate strength, the dimensions of the cutouts vary based on the statistical distribution boundaries, $1800 \leq h \leq 2900$ and $600 \leq b \leq 1100$, as illustrated in Section 3.2. For the selection of parameters, DOE is applied, using the CCD method. Four cases of the maximum diameter varying from $2750 \mathrm{~mm}$ to $4250 \mathrm{~mm}$ are noted with the locations of the cutouts in the vertical and circumferential directions, $h_{o} / H_{S}=$ 0.1 and $\theta=0$ degree. A total of 1,080 cases of series analyses are performed, and the parameters considered are as follows:

- Shape: rectangular, elliptical, half-rectangular-elliptical

- Height of the cutout, $h: 1800 \sim 2900 \mathrm{~mm}$

- Width of the cutout, $b: 600 \sim 1100 \mathrm{~mm}$

- Diameter, $D: 2750,3250,3750,4250 \mathrm{~mm}$

- Column slenderness ratio, $\lambda=D / t: 90,110,125,130,150$

- Loading condition: axial compression and pure bending

The volume of the cutout is defined as:

$$
V=A t
$$

where $V$ is the volume of the cutout, $A$ is its area and $t$ is the thickness of the wind turbine tower. It is assumed that the change in the breadth of the cutout due to curvature 
is ignored to simplify the calculation of the cutout area.

Figs. 23 and 24 show an example of the effect of column slenderness and aspect ratios on the non-dimensionalised ultimate strength $(D=3750 \mathrm{~mm})$ for 3 shapes under axial compression and pure bending. It is found that as the height of the cutout, $h$ increases for the same breadth, $b$, the ultimate strength clearly decreases. It is observed that as the volume of the cutout increases for the same aspect ratio, the strength decreases due to increasing thickness, yet the reduced ultimate strength appears nearly the same regardless of the cutout shape.

Figs 25 and 26 illustrate the non-dimensionalised ultimate strength of selected design points for the 3 cutout shapes under axial compression and pure bending. It is found that the ultimate strength reduction appears within a range of 50-80\% of the reference strengths for both loading conditions.

\section{Empirical Formulation of the Ultimate Strength}

The results of the parametric analysis described in Section 5.3 are used to derive empirical formulations that can predict the ultimate strength of a circular cylindrical shell with the cutout. The linear regression approach with the least-squares method is used, as follows:

$$
\begin{aligned}
& F_{u} / F_{R}=\xi_{D} D_{\text {max }}+\xi_{t} t+\xi_{h} h+\xi_{b} b+\xi_{C} \\
& M_{u} / M_{P}=\zeta_{D} D_{\text {max }}+\zeta_{t} t+\zeta_{h} h+\zeta_{b} b+\zeta_{C}
\end{aligned}
$$

where $F_{u} / F_{R}$ and $M_{u} / M_{P}$ are the non-dimensionalised ultimate strengths under axial compression and pure bending, respectively. The coefficients for axial compression and

pure bending are indicated in Table 4. The regression statistics, including the correlation coefficients and the adjusted R-square $\left(R^{2}\right)$, are illustrated in Table 5. 
The correlation between the numerical results and the empirical estimations of the ultimate strength is illustrated in Fig. 27. It is found that the estimations made by the proposed empirical equations well agree with the numerical calculations. This implies that the proposed empirical equations can be an effective way to estimate the reduced ultimate strength of circular cylindrical shells with cutouts.

The design formulae developed in the present study cover an extensive range of possible geometrical variations in circular cylindrical shells with cutouts, and thus they would provide guidance for wind turbine tower design. It should be cautioned that they may need to be validated further by comparison with more accurate computations and experiments before being used in special cases with geometric and boundary conditions.

\section{Conclusion}

The aims of this study were to develop a numerical modelling technique that accurately predicts structural responses by considering nonlinearities, and to numerically examine the effects of various variables on the ultimate-strength characteristics of wind turbine towers with cutouts. A series of nonlinear finite element computations were undertaken to achieve these objectives, and several conclusions can be drawn from the results, as outlined below.

(1) First, the wind turbine structures in service were investigated, and their actual dimensional characteristics and those of their cutouts were identified from the data collected and analysed.

(2) A nonlinear finite element modelling technique was developed based on a mesh convergence study and validation studies for wind turbine towers with cutouts.

(3) It was confirmed that the effect of the cutout shape is negligible, whereas placing the cutout on the compression side produced the minimum ultimate 
strength. Further, under pure bending, the ultimate strength appeared to be uniform when the cutout angle was over 90 degrees.

(4) Based on the results of a parametric analysis, a design formula accommodating a whole range of actual dimensional characteristics is proposed. This formula has the potential to improve the design and safety assessment of circular cylindrical shells with cutouts.

(5) Given the numerous uncertainties involved due to geometrical and boundary conditions, among others, further studies are recommended to examine models used to examine the collapse mechanism more realistically.

\title{
Acknowledgements
}

This research was supported by Basic Science Research Program through the National Research Foundation of Korea (NRF) funded by the Ministry of Education (NRF-2014R1A1A2006102, NRF-2015R1A6A3A01060166 and NRF-20XX).

\section{Appendix A.}

\author{
A.1. Tables
}

\section{References}

ANSYS, 2015. User's manual (version 16.1). ANSYS Inc., Canonsburg, PA, USA.

Azizian Z.G. and Roberts T.M., 1983, Buckling and elasto-plastic collapse of perforated plates, In Proceedings of the International Conference on Instability and Plastic Collapse of Steel Structures, Granada Publishing, London, UK, pp. 392-398.

Betten J. and Shin C.H., 2000, Elasto-plastic buckling analysis of rectangular plates subjected to biaxial loads, Forschung im Ingenieurwesen, Vol. 65, pp. 273-278.

Brazier L.G., 1927, On the flexure of thin cylindrical shells and other "thin" sections. Proceedings of Royal Society of London, Series A. Vol. 116(773), pp.104-114, DOI: $10.1098 /$ rspa.1927.0125. 
Brown C.J. and Yettram A.L., 1986, The elastic stability of square perforated plates under combinations of bending, shear and direct load, Thin-Walled Structures, Vol. 4(3), pp.239-246.

Collu M., Brennan F.P. and Patel M.H., 2014, Conceptual design of floating support structure for an offshore vertical axis wind turbine: the lessons learnt, Ships and Offshore Structures, Vol. 9(1), pp.3-21.

Dimopoulos C.A. and Gantes C.J., 2012, Experimental investigation of buckling of wind turbine tower cylindrical shells with opening and stiffening under bending, Thin-Walled Structures, Vol. 54, pp.140-155.

DIN 18800-4, 1990, Stahlbauten: Stabilitätsfälle, Schalenbeulen, Beuth Verlag, Berlin, Germany.

DNVGL, 2013a, Design of offshore wind turbine structures, Report No.: DNV-OS-J101, Det Norske Veritas, Oslo, Norway.

DNVGL, 2013b, Buckling strength of shells, DNV-RP-C202, Det Norske Veritas, Oslo, Norway.

Durban D. and Zuckerman Z., 1999, Elasto-plastic buckling of rectangular plates in biaxial compression/tension, International Journal of Mechanical Science, Vol. 41, pp. 751-765.

ECCS, 1980, Technical Committee 8, Structural Stability, TWG 8.4-Shells, Buckling of Steel Shells, European Design Recommendations, 1st edition.

El-Sawy K.M., Nazmy A.S. and Martini M.I., 2004, Elasto-plastic buckling of perforated plates under uniaxial compression, Thin-Walled Structures, Vol. 42, pp.1083-1101.

EN1993-1.6, 2006, European Committee for Standardization, Eurocode 3: Design of Steel Structures, Part 1-6: Strength and Stability of Shell Structures.

EWEA, 2014, Wind in power, 2014 European statistics, European Wind Energy Association, Brussels, Belgium, Available online: http://www.ewea.org/ fileadmin/files/library/publications/statistics/EWEA-Annual-Statistics-2014.pdf.

Fabian O., 1977, Collapse of cylindrical, elastic tubes under combined bending, pressure and axial loads, International Journal of Solids Structures, Vol. 13, pp. 1257-1270.

Gellin S., 1980, The plastic buckling of long cylindrical shell under pure bending, International Journal of Solids Structures, Vol.16, pp.397-407. 
Ghanbari Ghazijahani T., Jiao H. and Holloway D., 2015, Structural behaviour of shells with different cutouts under compression: An experimental study, Journal of Construction Steel Research, Vol. 105, pp.129-137.

Guo L., Yang S. and Jiao H., Behaviour of thin-walled circular hollow section tubes subjected to bending, Thin-Walled Structures, Vol. 73, pp. 281-289.

Han H., Cheng J., Taheri F. and Pegg N., 2006, Numerical and experimental investigations of theresponse of aluminum cylinders with a cutout subject to axial compression, Thin-Walled Structure, Vol.44, pp.254-270.

Jensen F.M., Falzon B.G., Ankersen J. and Stang H., 2006, Structural testing and numerical simulation of $34 \mathrm{~m}$ composite wind turbine blade, Composite Structures, Vol. 76, pp. 52-61.

Ju G.T. and Kyriakides S., 1992, Bifurcation and localization instabilities in cylindrical shells under bending II. Predictions, International Journal of Solids Structures, Vol. 29, pp. 1143-1171.

Jullien J. and Limam A., 1998, Effects of openings of the buckling of cylindrical shells subjected to axial compression, Thin-Walled Structure, Vol.31, pp.187-202.

Kim U.N., Choe I.H. and Paik J.K., 2009, Buckling and ultimate strength of perforated plate panels subject to axial compression: experimental and numerical investigations with design formulations, Ships and Offshore Structures, Vol.4(4), pp. 337-361.

Kyriakides S. and Ju G.T., 1992, Bifurcation and localization instabilities in cylindrical shells under bending. I. Experiments, International Journal of Solids Structures, Vol. 29, pp. 1117-1142.

Kühlmeier L., 2007, Buckling of wind turbine rotor blades. Analysis, design and experimental validation, $\mathrm{PhD}$ thesis, Aalborg University, Denmark, ISBN: 8791464-00-5.

Lee H.G. and Park J., 2016, Static test until structural collapse after fatigue testing of a full-scale wind turbine blade, Composite Structures, Vol. 136, pp. 251-257.

Lee S.E., Paik J.K., Ha Y.C., Kim B.J. and Seo J.K., 2014, An efficient design methodology for subsea manifold piping systems based on parametric studies, Ocean Engineeing, Vol. 84, pp. 273-282.

Overgaard L.C.T., Lund E. and Camanho P.P., 2010a, A methodology for the structural analysis of composite wind turbine blades under geometric and material induced instabilities, Composite Structures, Vol.88, pp.1092-1109. 
Overgaard L.C.T., Lund E. and Thomsen O.T., 2010b, Structural collapse of a wind turbine blade, Part A: static test and equivalent single layered models. Composites Part A: Applied Science and Manufacturing, Vol. 41, pp. 257-270.

Overgaard L.C.T. and Lund E., 2010, Structural collapse of a wind turbine blade, Part B: progressive interlaminar failure models, Composites Part A: Applied Science and Manufacturing, Vol.41, pp. 271-283.

Paik J.K., 2007, Ultimate strength of steel plates with a single circular hole under axial compressive loading along short edges, Ships and Offshore Structures, Vol. 2(4), pp.355-360.

Reissner E., 1961, On finite pure bending of cylindrical tubes, Österreichisches Ingenieur-Archiv, Vol.15, pp.165-172.

Sabir A.B. and Chow F.Y., 1983, Elastic buckling of flat panels containing circular and square holes, In Proceedings of the International Conference on Instability and Plastic Collapse of Steel Structures, Granada Publishing, London, UK, pp. 311321.

Sahin S., 2016, Wind turbine tower structures analysis according to wind load in terms of cost, Master thesis, University of Liege.

Schenk C.A. and Schuëller G.I., 2007, Buckling analysis of cylindrical shells with cutouts including random boundary and geometric imperfections, Cimputer Methods in Applied Mechanics and Engineering, Vol.196, pp.3424-3434.

Seide P. and Weingarten V.I., 1961, On the buckling of circular cylindrical shells under pure bending, Journal of Applied Mechanics, Vol. 28, pp.112-116.

Shangmugam N.E., Thevendran V. and Tan Y.H., 1999, Design formula for axially compressed perforated plates, Thin-Walled Structures, Vol. 34(1), pp. 1-20.

Shariati M. and Rokhi M.M., 2008, Numerical and experimental investigations on buckling of steel cylindrical shells with elliptical cutout subject to axial compression, Thin-Walled Structure,Vol.46, pp.1251-1261.

Shariati M. and Rokhi M.M., 2010, Buckling of steel cylindrical shells with an elliptical cutout, International Journal of Steel Structures, Vol.10, pp.193-205.

Sherman D.R., 1976, Tests of circular steel tubes in bending, ASCE Journal of the Structural Division, Vol. 102, pp.2181-2195.

Tennyson R.C., 1968, The effects of unreinforced circular cutouts on the buckling of circular cylindrical shells under axial compression, Journal of Engineering for Industry, Vol.90(4), pp.541-546. 
Wang G., Sun H., Peng H. and Uemori R., 2009, Buckling and ultimate strength of plates with openings, Ships and Offshore Structures, Vol. 4(1), pp.43-53.

Yang J., Peng C., Xiao J., Zeng J., Xing S. and Jin J., 2013, Structural investigation of composite wind turbine blade considering structural collapse in full-scale static tests, Composite Structures, Vol. 97, pp. 15-29.

Yeh M.K., Lin M.C., and Wu W.T., 1999, Bending Buckling of an Elasto-plastic Cylindrical Shell with a Cutout, Engineering Structures, Vol. 21, pp.996-1005. 